\title{
¿Hacia el docuketing? Una experiencia de colaboración docente en el grado de Publicidad y Relaciones Públicas de la Universitat Autònoma de Barcelona
}

Towards "docuketig"? A collaborative teaching experience in the pregraduate studies of Advertisement and Public Relations of the Universitat Autònoma de Barcelona

\author{
Alfons GonzÁlez Quesada (1) y Joan Ramon Molero I YLL $(1,2)$ \\ (1) Facultat de Ciències de la Comunicació, Universitat Autònoma de Barcelona, España \\ (2) EUSS Enginyeria, Escola Universitària Salesiana de Sarrià, Barcelona, España
}

(1) Alfons.Gonzalez@uab.cat (2) jrmolero@euss.cat

\begin{abstract}
Resumen
La adaptación de los planes de estudio a las exigencias impuestas por el Espacio Europeo de Educación Superior ha brindado una gran oportunidad a la innovación docente. La presente contribución expone los valoración de la puesta en marcha de una asignatura singular en el grado de Publicidad y Relaciones Públicas de la Universidad Autónoma de Barcelona. Marketing y Gestión Documental, concebida como una asignatura en la que debían colaborar dos disciplinas, ha supuesto un reto para potenciar el trabajo cooperativo entre equipos docentes y aumentar la visibilidad de la documentación, tanto entre los estudiantes del grado como entre el resto de docentes en el ámbito de la comunicación.
\end{abstract}

Palabras clave: Mercadotecnia. Biblioteconomía y Documentación. Interdisciplinariedad. Grado de Publicidad y Relaciones Públicas. Espacio Europeo de Educación Superior.

\section{Introducción}

En el curso 2010-2011, la Facultad de Ciencias de la Comunicación de la Universitat Autònoma de Barcelona (UAB) puso en marcha los planes de estudios de los Grados de Periodismo, Comunicación Audiovisual y Publicidad y Relaciones Públicas. Ello supuso el encargo docente de nuevas materias y la aplicación de metodologías adecuadas a las exigencias del Espacio Europeo de Educación Superior.

Entre esas nuevas materias destaca Proyectos, considerada obligatoria y desarrollada en los tres primeros cursos del grado de Publicidad y Relaciones Públicas. Esta materia debe imbricar el trabajo conjunto de diversas disciplinas en la consecución de objetivos comunes. En el momento de elaboración de los nuevos planes de estudio los Proyectos representaban una apuesta por la innovación y la voluntad de proporcio-

\begin{abstract}
The adaptation of curricula to the requirements imposed by the European Higher Education Area has provided a great opportunity for innovation in teaching. This contribution presents the assessment of the implementation of a singular subject in the Advertising and Public Relations degree at the Autonomous University of Barcelona. Marketing and Document Management, conceived as a subject in which two disciplines must work together has been a challenge to enhance collaborative work between teams of teachers and increase the visibility of information science, both among students and between teachers in the field of communication.
\end{abstract}

Keywords: Marketing. Information and Library Science. Advertising and Public Relations degree. Interdisciplinarity. European Higher Education Area.

nar un valor añadido a todos los grados, que podía actuar también como un elemento diferencial y de referencia respecto de la oferta de otras facultades de comunicación. En las primeras fases de la elaboración de los planes de estudio, los tres nuevos grados contaban con Proyectos en sus tres primeros cursos, pero la dinámica de la elaboración de los planes estuvo dominada por imperativos ajenos a los estrictamente académicos, $y$, entre otros cambios, esa dinámica redujo la presencia de los Proyectos al grado de Publicidad. Es en esa 'isla' donde sobrevive la documentación en la Facultad de Ciencias de la Comunicación de la UAB, concretamente a través de la asignatura de primer curso Marketing y Gestión Documental. De esta manera, el grado de Publicidad es el único de la citada facultad que cuenta con una asignatura que permita adquirir las competencias y habilidades propias de la documentación. 


\section{Características de la asignatura}

Marketing y Gestión Documental forma parte de la materia Proyectos junto a otras dos asignaturas: Estrategias en Publicidad y Relaciones Públicas y Creatividad en Publicidad y Relaciones Públicas. Es una materia obligatoria con 36 créditos ECTS, a impartir en los tres primeros cursos del grado. Concretamente, el plan de estudios señala en relación a los Proyectos que deben proporcionar (ANECA, 2009).

los conocimientos teórico-prácticos aplicados a la resolución de casos reales, en el ámbito de la Publicidad y de las Relaciones Públicas, que se trabajarán a lo largo del curso. Incluye también los conocimientos teórico-prácticos básicos orientados al desarrollo de las habilidades de gestión documental en los procesos de captación, jerarquización, selección y uso discriminado de la información.

Marketing y Gestión Documental es una asignatura anual de 12 créditos ECTS, con un alto componente práctico, ya que la mitad de las actividades formativas dirigidas tienen ese carácter, mientras que poco más del $10 \%$ son teóricas. Como se desprende de su denominación, esta asignatura incluye contenidos de dos disciplinas, dualidad reflejada lógicamente en sus objetivos formativos:

- Saber identificar mercados y públicos.

- Comprender el alcance de las herramientas y recursos básicos en el marketing analítico y saber aplicarlos.

- Saber usar las principales tipologías de fuentes y recursos de información.

- Saber gestionar la información documental.

Es importante también atender al itinerario formativo que seguirá el estudiante tras su paso por esta asignatura, ya que sólo tendrá continuidad en relación al componente de marketing, en tercer curso con la asignatura Marketing estratégico y operativo.

\section{La experiencia previa: Marketing y Gestión documental en la antigua licenciatura}

En el plan de estudios de la licenciatura en Publicidad y Relaciones Públicas de 1992, los dos contenidos que configuran la asignatura del grado ya estaban presentes, pero a través de más de una asignatura. En el caso del ámbito documental a través de una troncal, Documentación informativa, impartida en el segundo semestre del segundo año de licenciatura. Su objetivo era capacitar a los estudiantes en el uso adecuado de los principales tipos de fuentes de información, así como en el manejo de los recursos específicos más útiles para la práctica publicitaria. En cuarto curso existía una asignatura optativa, Informática documental y teledocumentación, concebida para capacitar a los estudiantes en el diseño y evaluación de bases de datos documentales. Ambas asignaturas compartían una orientación eminentemente práctica. Por lo que respecta al ámbito marketing, los alumnos tenían el primer contacto con sus contenidos en el segundo curso, mediante Introducción a la Mercadotecnia. Se trataba de una asignatura anual, que permitía abordar ampliamente todos los aspectos del marketing analítico, estratégico y operativo.

\section{Organización de la docencia}

Las áreas de marketing y de documentación pertenecen a departamentos distintos, con dinámicas de trabajo diferentes. Desde las direcciones de ambos departamentos (Publicidad, Relaciones Públicas y Comunicación Audiovisual y el Área de Documentación del Departamento de Filología Catalana) se designó a los profesores Joan Ramon Molero y Alfonso González como responsables de la asignatura y coordinadores del resto del equipo docente que debería intervenir en la impartición de la asignatura. La necesidad de relacionar dos ámbitos, hasta ese momento tan ajenos, en un proyecto común ha exigido un alto grado de coordinación.

El primer paso para elaborar la programación de la asignatura fue el conocimiento mutuo de los contenidos, que hasta entonces se habían impartido en la licenciatura, así como el intercambio de experiencias docentes y de evaluación. Ninguno de los coordinadores era ajeno al hecho de que los puntos de encuentro, a tenor de los contenidos de ambas disciplinas, eran prácticamente nulos, y que la necesidad de imbricarlos no debía desnaturalizar ninguna de las asignaturas previas. Se decidió que las sesiones de marketing y documentación se alternasen semanalmente. Cualquier otra solución, como repartir un semestre para cada ámbito, hubiera anulado el propósito inicial de la materia, además de proyectar una imagen contraria a la de trabajar en pos de objetivos comunes. También se decidió, de acuerdo al peso de las actividades formativas recogidas en el plan de estudios, que de las tres horas de actividades dirigidas de cada sesión, dos fuesen prácticas y la otra de exposición teórica. Esta fue la organización docente del primer semestre.

En el segundo la dinámica varió significativamente. Se mantuvo la alternancia semanal y el 
mismo peso de las actividades prácticas dirigidas. El lugar de las exposiciones teóricas lo ocuparon tutorías grupales, programadas para orientar y ayudar a los estudiantes en la resolución de un caso. De esta manera, el trabajo grupal ha sido el núcleo de la actividad del segundo semestre, trabajo en el que los estudiantes han tenido que aplicar y desarrollar de manera práctica las competencias y habilidades adquiridas en marketing y gestión documental.

El trabajo grupal también ha representado el punto de encuentro de los dos componentes de la asignatura. Su objetivo ha sido aproximar la dinámica del marketing y la gestión documental a la actividad profesional publicitaria mediante la resolución de un caso: la simulación de un encargo al que un equipo de publicitarios ha debido dar respuesta. El encargo ha girado en torno al diseño de una campaña de sensibilización social, pero la función última del trabajo no era diseñar la campaña, sino dar los primeros pasos que conducirían a su realización, a través de la creación de un caso práctico sobre el tema propuesto y la obtención de la documentación necesaria que serviría para conocer a fondo el tema.

De esta manera, cada equipo ha buscado y seleccionado la información necesaria en base a un itinerario formativo establecido previamente por el docente de acuerdo al perfil de su caso. Los estudiantes han accedido a las fuentes que fundamenten el caso práctico (la campaña) y las decisiones que se tuvieran que tomar en el futuro (creación y producción).

El resultado de estos procesos se ha concretado en un informe expuesto públicamente y que resume el caso y la documentación imprescindible. A través del trabajo grupal y de su seguimiento en las sesiones de tutoría, los estudiantes han percibido la relación que se establece entre los dos componentes de la asignatura.

\section{Requerimientos tecnológicos y de infraestructura y equipo docente}

La dimensión eminentemente práctica de la asignatura y la metodología docente aplicada han exigido contar con diversos tipos de espacios. En las exposiciones teóricas se ha utilizado un aula convencional dotada de equipamiento informático y audiovisual con capacidad para 80-90 alumnos. Cuando la clase se dividía para trabajar por equipos los contenidos de marketing se utilizaron dos seminarios, mientras que las sesiones prácticas de gestión documental se realizaron en cuatro aulas informatizadas.
El equipo docente ha constado de seis profesores: dos del área de marketing y cuatro del área de documentación. De esta manera, en las sesiones prácticas, la distribución de alumnos por docente variaba. En el caso de marketing eran 40 alumnos por profesor, mientras que en las sesiones prácticas de gestión documental la ratio se situaba en 20 alumnos.

\section{Perfil de los estudiantes}

En la primera sesión pedimos a los estudiantes que contestasen a un pequeño formulario $\mathrm{Wel}-$ come-quiz. La información obtenida nos permitió trazar el perfil general de los estudiantes. Entre los datos más relevante destacaron:

- El elevado número de estudiantes procedentes de Ciclos Formativos de Grado Superior, cercano al $25 \%$.

- El número de mujeres doblaba con creces al de hombres.

- Más de la mitad tenían o habían tenido algún tipo de experiencia laboral.

- Sólo 2 de los 82 alumnos iniciales habían tenido algún tipo de contacto profesional con el mundo de la gestión documental, mientras que 24 , más de una cuarta parte, lo había tenido con el del marketing o la comunicación social.

Este último dato no carece de relevancia, porque entre los estudiantes existía un desconocimiento absoluto sobre la realidad profesional vinculada a la gestión de la información, lo que se traduce en una muy baja visibilidad social.

\section{Metodología docente}

Desde el primer momento se ha procurado ceder el protagonismo al estudiante a través de una metodología activa, tanto en las sesiones teóricas, como en las actividades prácticas dirigidas. El objetivo ha sido conseguir que el estudiante participe en clase y se haga coresponsable de su formación. Para ello se ha procurado diseñar situaciones de aprendizaje en las que los alumnos adquiriesen las competencias y habilidades adecuadas.

La docencia de contenidos teóricos se ha basado en el método expositivo. A pesar de que este método despierte recelos por considerarse que puede inducir a una actitud pasiva del estudiante, las clases magistrales han fomentado su participación, bien mediante las preguntas que los alumnos han formulado al docente, o por las que el profesor ha dirigido a los estudiantes. En el caso de marketing, las sesiones teóricas han

González Quesada, Alfons; Molero i YII, Joan Ramon,. ¿Hacia el docuketing? Una experiencia de colaboración docente en el grado de Publicidad y Relaciones Públicas de la Universitat Autònoma de Barcelona // 17:2 (jul.-dic. 2011) 81-85. ISSN 1135-3716. 
servido para exponer los contenidos teóricos a través de la presentación de ejemplos extraídos de la práctica profesional. En el ámbito documental, este tipo de sesiones han incidido especialmente en dar a conocer el mundo de las fuentes de información y su uso, también a través de ejemplos aplicados a la práctica investigadora o profesional.

Las actividades prácticas dirigidas han sido el escenario idóneo para aplicar la técnica del Aprendizaje Basado en Problemas, como punto de partida para la adquisición e integración de nuevos conocimientos. Esta dinámica de trabajo ha potenciado el protagonismo de los estudiantes en el proceso de aprendizaje, y ha sentado las bases teóricas y prácticas, para la realización posterior de la resolución del caso a través del trabajo grupal.

Las tutorías, puestas en marcha en el segundo semestre, han garantizado un seguimiento constante del trabajo grupal. Los alumnos han tenido una autonomía relativa en la realización de su trabajo (cada equipo daba respuesta a un caso distinto), ya que este estaba determinado por un itinerario previo, pautado por el docente y que aseguraba así la consecución de objetivos comunes para todos los estudiantes. El trabajo en equipo, como se ha puesto de manifiesto previamente, ha constituido un instrumento esencial para fomentar el trabajo colaborativo. Las tutorías también han potenciado la polivalencia de los docentes, ya que su función no se ha limitado a la exposición de contenidos teóricos. El conjunto del equipo docente ha acompañado a los estudiantes en su proceso de adquisición de competencias y ha actuado como facilitador de su aprendizaje.

Otro de los aspectos fundamentales que han guiado la labor del equipo docente fue fomentar entre los alumnos la percepción de que Marketing y Gestión documental no se trataba de dos asignaturas independientes que compartían un marco horario, sino de una asignatura unitaria en la que confluían dos disciplinas en la consecución de objetivos comunes. Tal percepción se fomentó mediante diversas estrategias, como la citada alternacia de las sesiones de marketing y documentación, o la realización de sesiones conjuntas con la participación de profesores de los dos ámbitos. Otros elementos que han ayudado a fortalecer esa percepción unitaria ha sido la elaboración conjunta de guías didácticas para los estudiantes, e incluso la realización en una misma sesión de la prueba de evaluación escrita, con un único enunciado incluyendo cuestiones de ambas disciplinas.

\section{Evaluación}

Para evaluar el grado de adquisición de conocimientos planteados en el curso se han valorado cinco elementos. El trabajo desarrollado en ellos ha configurado el portafolio del alumno. Cada uno de estos elementos ha tenido un valor distinto.

- Prácticas individuales de aula. Realizadas durante el primer semestre. Concebidas para mostrar los procesos y herramientas que deberían utilizar los estudiantes en el desarrollo del trabajo en equipo. Se realizaron 14 ejercicios prácticos, 7 para cada ámbito de la asignatura. El valor que podían llegar a tener en la nota final de la asignatura era del $20 \%$. La nota media obtenida por los estudiantes que siguieron la evaluación continua fue de 1 '95 sobre 2, lo que equivale a una cualificación excelente y a un altísimo nivel de asistencia a las sesiones prácticas.

- Prueba escrita. Realizada a mediados del segundo semestre con el objetivo de evaluar el nivel de asimilación de los contenidos teórico-prácticos de los dos componentes de la asignatura. El valor que podía llegar a tener en la nota final de la asignatura era del $20 \%$. La calificación media obtenida por los estudiantes que siguieron la evaluación continua fue de 1'14 sobre 2 . Se trata de un resultado muy discreto.

- Informe individual de evaluación. Este ejercicio consistía en la elección por parte del alumno de los tres aspectos de los contenidos de cada ámbito de la asignatura que había considerado más importantes. En el informe debía justificar su elección y destacar qué había aprendido mediante tales contenidos. La elección se efectuó el mismo día en que se realizó la prueba escrita y los alumnos tuvieron cerca de un mes para elaborar su informe. El valor que podía llegar a tener este ejercicio en la nota final era del $10 \%$. La calificación media obtenida por los estudiantes que siguieron la evaluación continua fue de 0,85 sobre 1 . Se trata de una calificación óptima.

- Trabajo grupal consistente en el planteamiento y resolución de un caso. Se realizó durante el segundo semestre e integró en la práctica los contenidos trabajados en el primer semestre. La dinámica del trabajo en equipo debía ayudar a consolidar la adquisición de las competencias y habilidades desarrolladas previamente de manera individual. El valor que podía llegar a tener el trabajo en 
la nota final era del $40 \%$. La calificación media obtenida por los estudiantes que siguieron la evaluación continua fue de 3,38 sobre 4. Se trata de un resultado notable.

- Otras evidencias del aprendizaje. Se trata de un conjunto de ejercicios de realización voluntaria. El valor que podían llegar a tener en la nota final era del $10 \%$, sólo en el caso de que el resto de ítems de la evaluación continua se hubieran aprobado. La calificación media obtenida por los estudiantes que hicieron alguna tarea voluntaria fue de 0,27 sobre 1 , lo que demuestra un bajo nivel de contribuciones, especialmente en el ámbito de marketing.

A tenor de lo expuesto, la calificación media global fue 7,99 entre los 71 estudiantes que superaron la asignatura. De los 82 alumnos iniciales, siete la suspendieron y el resto fueron abandonos. A pesar de que la calificación media fue muy notable, el equipo docente ha detectado una serie de disfunciones en el sistema de evaluación y ha propuesto una serie de medidas correctoras que se comentarán más adelante.

\section{Conclusiones}

La valoración global de la puesta en marcha de una asignatura con las peculiaridades de Marketing y Gestión Documental ha sido altamente positiva. Los resultados académicos obtenidos por los estudiantes y la valoración que estos han dado a la tarea docente del equipo de profesores ha sido, en ambos casos, notable. Sin embargo, se han detectado ciertas disfunciones en el funcionamiento de la asignatura que han obligado a sus coordinadores a proponer una serie de cambios que se han implementado en el curso académico 2011-12.

Algunos de estos cambios han afectado al valor porcentual de algunas actividades formativas para adecuarlo al esfuerzo dedicado por los estudiantes en su realización. Otros tienen que ver con la reordenación de ciertas actividades de evaluación.

Así, el informe individual de evaluación será previo a la realización de la prueba escrita, con objeto de que ese informe sea un instrumento útil para los estudiantes a la hora de preparar la prueba escrita. De esta manera se busca au- mentar el rendimiento académico de esa prueba.

También se ha creído oportuno potenciar la evaluación continuada del trabajo grupal, de manera que el peso de la valoración no recaiga básicamente en la parte final (presentación del informe y exposición), sino que existan evidencias del aprendizaje recogidas en las diferentes tutorías. Para ello se contará con rúbricas que permitan valorar una serie de parámetros de acuerdo al itinerario formativo previsto para cada sesión.

También en relación con el trabajo grupal, hasta ahora la propuesta del caso procedía del equipo docente, a modo de encargo profesional, ahora la propuesta del caso será formulada por los estudiantes y el equipo docente la validará antes de fijar su itinerario formativo. Con este cambio se pretende obtener un mayor implicación en el trabajo, ya que el caso propuesto surgirá del consenso de cada equipo. De esta manera también se busca potenciar desde mucho antes la dinámica grupal y el trabajo cooperativo.

Para abundar en la dimensión colaborativa del trabajo grupal, una parte de su realización se hará a través de la wiki del campus virtual de la UAB.

Por último, las actividades voluntarias, que deben proporcionar el plus de excelencia en la calificación de los estudiantes, se fijarán con más precisión en el calendario de la asignatura. Algunas de estas actividades, vinculadas al ámbito documental, se realizarán conjuntamente con otras asignaturas del grado, con el objetivo de potenciar tanto la colaboración docente, como la percepción de la aplicabilidad de los contenidos de la gestión documental en el proceso del trabajo académico y en el aprendizaje de otras materias del grado. Con ello también se busca recuperar parte de la visibilidad que la disciplina documental ha perdido en la Facultad de Ciencias de la Comunicación de la Universitat Autònoma de Barcelona.

\section{Referencias}

ANECA. Grado de Publicidad y Relaciones Públicas. Universitat Autònoma de Barcelona, 2009. 
